\title{
Repeated Shadow Track Orbits for Space-SunSetter Missions
}

\author{
Ahmed Gad and Ossama Abdelkhalik \\ Department of Mechanical Engineering-Engineering Mechanics, Michigan Tech University, 815 R.L. Smith Bldg, \\ 1400 Townsend Dr., Houghton, MI 49931, USA \\ Correspondence should be addressed to Ahmed Gad, ahgadels@mtu.edu
}

Received 14 May 2009; Accepted 10 September 2009

Recommended by Tal Shima

This paper introduces a new set of orbits, the "Repeated Shadow Track Orbits." In these orbits, the shadow of a spacecraft on the Earth visits the same locations periodically every desired number of days. The $J_{2}$ perturbation is utilized to synchronize the spacecraft shadow motion with both the Earth rotational motion and the Earth-Sun vector rotation. Motivation for the design of new shadow track orbits comes from the need to save energy. The general mathematical model to design a Repeated Shadow Track Orbit (RSTO) is presented within this paper. RSTOs' conditions are formulated and numerically solved. Results show the feasibility of RSTOs. An optimization process is also developed to maximize the shadow duration over a given site. A Genetic Algorithm (GA) technique is utilized for optimization.

Copyright ( 2009 A. Gad and O. Abdelkhalik. This is an open access article distributed under the Creative Commons Attribution License, which permits unrestricted use, distribution, and reproduction in any medium, provided the original work is properly cited.

\section{Introduction}

Extremely large space structures are proposed for solar sail and space-based solar power generation missions. Solar sails, the size of San Francisco, are proposed to collect momentum from the solar radiation, for deep space missions [1]. Solar Power Satellites (SPSs) are also large space structures orbiting the Earth. SPSs are proposed to generate electricity in space and transmit it to receivers on the Earth using either microwave or laser transmissions [2]. For the purpose of cooling the Earth, and thereby reducing the global warming, it is also suggested to put a huge number of satellites at the first Lagrangian point between the Earth and the Sun, to provide shade for the Earth [3].

A great numbers of studies were conducted by NASA and the Department of Energy (DOE) during the seventies of the last century on the feasibility of the SPSs concept. The Office of Technology Assessment evaluated these studies and did not recommend immediate action toward implementation due to the technical challenges and high cost of the proposed mission at that time [4]. In mid nineties, NASA took a fresh look at the concept; however they did not recommend, even discourage, further investments in this direction [5]. Yet, an active interest toward implementing this concept can be seen nationally [6] and internationally $[7,8]$.
Aiming at reducing the cost of the generated electricity from SPSs, in this paper it is suggested to have multiple objectives for the same SPSs. SPSs orbiting Earth can provide shadow on ground for the Earth cooling purposes, generate electricity using its huge solar arrays, and save energy devoted to air conditioning in the shaded areas. Nations from the warm regions may participate in the cost of an SPS to get its shadow, if it can be provided to them on a regular basis. More than $70 \%$ of the operational expenses, for many buildings and facilities in warm regions, is dedicated for air conditioning [9]. It has been observed that the main reason for these extremely high operational expenses is the direct solar rays from the Sun [9]. The SPSs will serve as a Space SunSetter for these regions participating in SPSs missions only. A Space SunSetter will significantly reduce the operational expenses of every building in the shaded area.

There are two concepts for Space SunSetter under investigation. The first is to design a huge spacecraft orbiting the Earth. The main challenge in this concept is to find an appropriate orbit satisfying a repeated shadow track over a specific region on Earth's surface. The second concept is a stationary Space SunSetter. This concept depends on the idea of Space Elevator $[10,11]$. The purpose of this paper is to investigate the feasibility of the first concept. In this 
paper, a preliminary mission analyzes was done to calculate a rough size for the Space SunSetter at different altitudes. Since this type of mission will require the Space SunSetter shadow on ground to revisit the same place repeatedly, the orbital elements are developed to have a shadow visits a certain location on the Earth's surface for a given number of days, this is called the repeated shadow track condition.

To find the orbit, among all repeated shadow track orbits, that has maximum duration time over a certain point on the Earth, an optimization tool was developed using a genetic algorithm (GA). Genetic algorithm is an optimization technique, based on the Darwinian principle of the survival of the fittest, that performs a stochastic search of initial conditions that maximize a given objective function [12]. Genetic algorithms have been used in literature to find solution for the problems of spacecraft rendezvous [13], orbit transfer [14], Ground Surveillance [15], space trajectory optimization [16], and in optimizing orbits in the 3D elliptic restricted three body problem [17].

\section{Mission Analyzes}

The main purpose of Space SunSetter is to provide eclipse "shadow" for a specific area on the Earth's surface. This section discusses the parameters that affect the size of the Space SunSetter, and analyzes the effects of these parameters on the size. These parameters are the area to be covered by shadow, and the Space SunSetter altitude above the given region. The Sun's direction relative to the spacecraft, which varies due to the Earth's rotation round the Sun, also affects the sizing of the Space SunSetter.

Three regions of solar eclipses result from the Space SunSetter blocking the light that travels to the Earth [18]. Figure 1 shows the basic eclipse geometry for the Space SunSetter. The complete shadow region is totally eclipsed by the Space SunSetter. The partial shadow region is only partially obscured by a portion of the Space SunSetter. While the annular shadow region is partially obscured by the whole body of the Space SunSetter. The type and the surface area of the covered regions depend on three key parameters: (1) the spacecraft altitude, (2) the spacecraft surface area, (3) and the location of the Sun relative to the spacecraft. The angle between the spacecraft position vector and the sun position vector defines the direction of Sun rays blocked by the Space SunSetter. This angle is the shadow angle $\theta_{\text {sh }}$ illustrated in Figure 2.

A simple geometrical method is developed to study the shadow characteristics created by a Space SunSetter orbiting the Earth. To carry out these geometrical calculations, the Earth is considered to be an ideal sphere with an average radius $6378 \mathrm{~km}$. The Sun's radius is $696000 \mathrm{~km}$, and the average distance between the Earth and Sun is $149599870 \mathrm{~km}$. An assumption has been made that the Sun's light rays are not parallel, as illustrated in Figure 1. Planner and spherical trigonometry, based on Figure 1, are used to conduct a preliminary sizing estimation for a Space SunSetter at different altitudes. The geometrical method is used to roughly estimate the sizes of the different shadow's regions
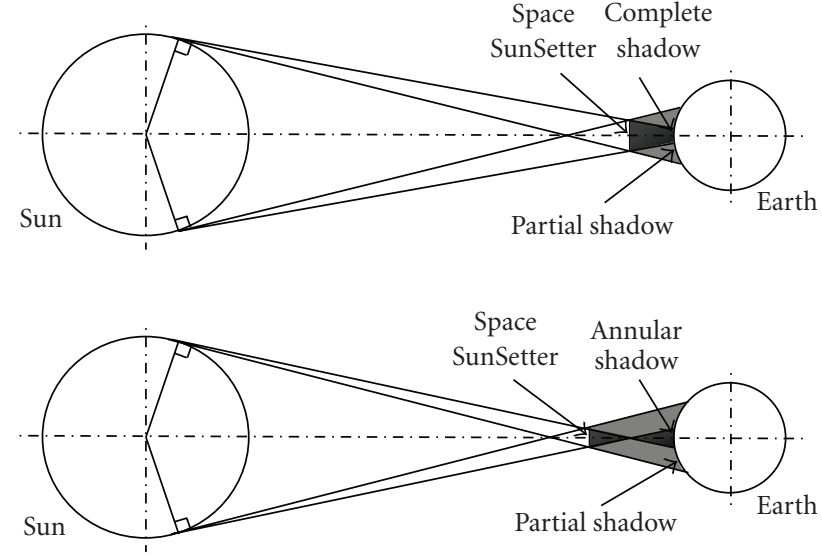

FIGURE 1: The general geometry for complete, annular, and partial shadow on the Earth's surface occurred by Space SunSetter. The figure is greatly exaggerated to illustrate the idea.

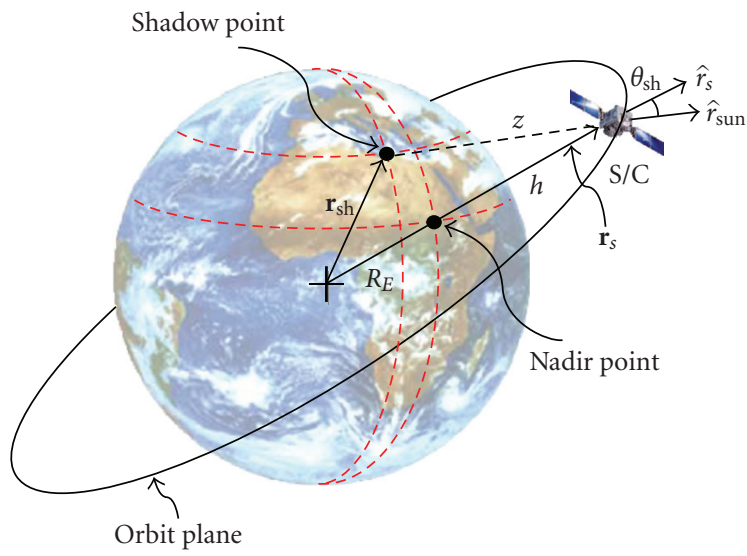

Figure 2: Shadow position vector, $\mathbf{r}_{\text {sh }}$, and shadow angle $\theta_{\text {sh }}$.

generated on the Earth at different altitudes and shadow angles. Figure 3(a) represents the surface area of Space SunSetter at different altitudes required to provide a complete shadow region on the Earth's surface with various radii $(1,5$, and $10 \mathrm{~km}$ ). Figure 3(b) shows the partial shadow area on the Earth's surface from the same Space SunSetter. The required surface area of a Space SunSetter increases with the spacecraft altitude to maintain complete shadow over a given area. Therefore, high Earth orbits (HEOs) are excluded because of the huge area requirements of a spacecraft at high altitudes.

Figure 4 shows the complete, partial, and annular shadow areas on ground created by a Space SunSetter with a radius of $5 \mathrm{~km}$, at different altitudes. The shadow angle is considered to be zero. The complete shadow region shrinks with increasing the altitude, vanishes at a certain altitude, then the annular shadow region develops. Although the annular shadow area increases with the Space SunSetter altitude, the eclipse percentage inside this region gradually decreases with the altitude. The area of the partial shadow region steadily increases with Space SunSetter altitude. Figures 5 and 6 show complete, partial, and annular shadow areas created by 


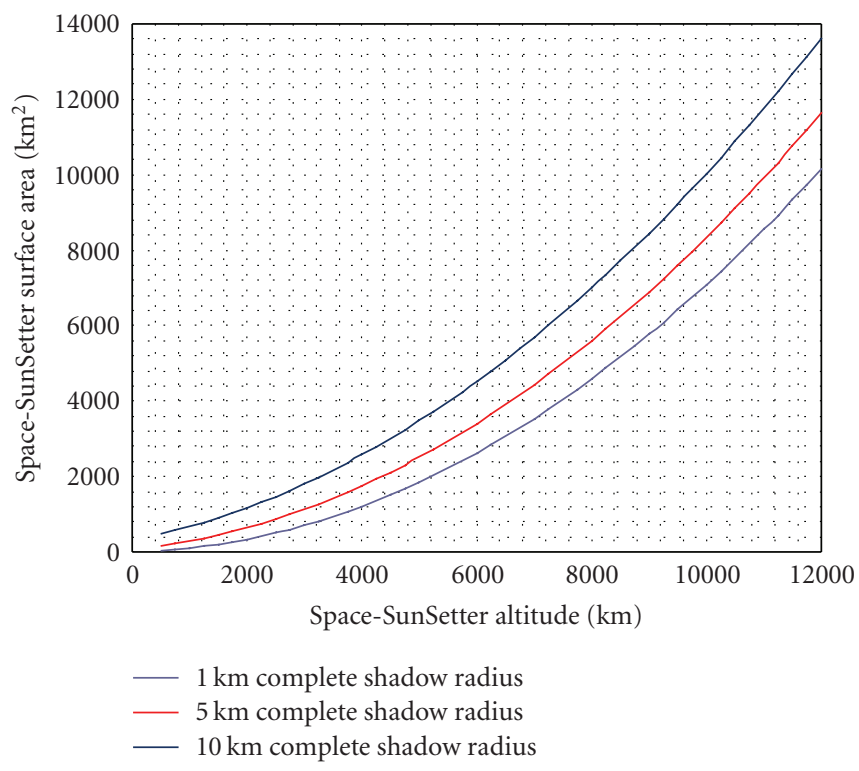

(a)

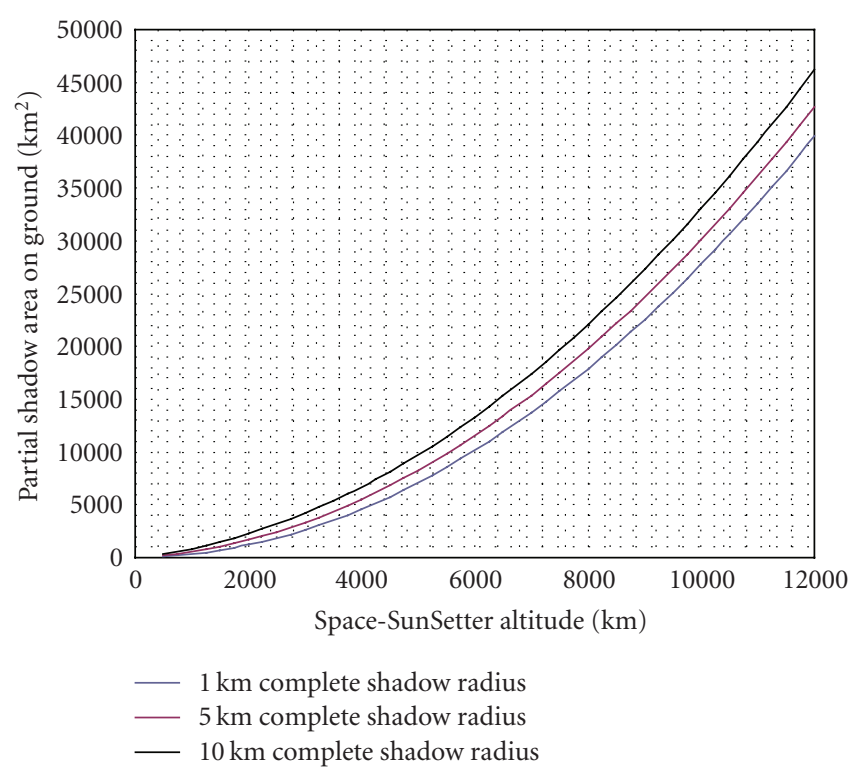

(b)

Figure 3: (a) Space SunSetter area, and its (b) partial shadow area on the Earth's surface.

a Space SunSetter with a radius of $5 \mathrm{~km}$ at altitude of 1000 and $1500 \mathrm{~km}$, respectively, at different shadow angles.

\section{Orbit Selection}

The well-known repeated ground track orbits have ground tracks that repeat every given number of days. The orbital elements may be selected such that the total change in the longitude of the nadir point, after an integer number of nodal periods, is an integer multiple of one complete Earth rotation about its axis [19]. The fact that we are using the nodal period results in no change in the nadir point latitude after any integer number of complete nodal periods. In the case of a repeated shadow track orbit, the Shadow-Nodal period is introduced. Shadow-Nodal period is the time it takes the shadow of a spacecraft to come back to the same latitude, after orbiting the Earth once.

In repeated shadow track orbits (RSTOs), the orbital elements, are selected such that the total change in the longitude of the shadow point, after an integer number of Shadow-Nodal periods, is an integer multiple of one complete Earth rotation about its axis. The orbit parameters selection of an RSTO is affected by the Earth's rotation rate around its axis as well as its rotation rate around the Sun.

At low altitudes, drag forces cannot be neglected for large cross-sectional area spacecraft [19]. In this analyzes, low Earth orbits (LEOs) are excluded, and hence aerodynamic drag is neglected. Since high Earth orbits (HEOs) are excluded due to size constrains (see Section 2), third body effect can be neglected. Moreover, the zonal harmonic coefficients (other than $J_{2}$ ) can be neglected due to its small values with respect to $J_{2}$ [19]. The resulting perturbing effect on the spacecraft's motion due to tesseral harmonics is small and periodic [19]. However, tesseral harmonics can produce moderate changes in the spacecraft's orbit if the orbit is in a resonant state with respect to the Earth's gravitational field (such as repeated ground track orbits) [19]. This paper deals with repeated shadow track orbits which are different from the repeated ground track orbits in the sense that they are not in resonance with respect to the Earth's gravitational field. Hence, the tesseral harmonics can be neglected in this analyzes. Solar radiation pressure (SRP) causes periodic variations in all of the orbit elements [18]. The SRP cannot be neglected, because of the large cross-sectional area of a Space SunSetter. However, in this preliminary analyzes, the SRP effect is neglected in the dynamic model. The SRP can be considered in the future studies.

3.1. Shadow Location Calculations. The spacecraft should be located on the line from the Earth to the Sun to obtain a shadow point on the Earth surface. The angle between the spacecraft position vector and the Sun position vector is defined as the shadow angle $\theta_{\mathrm{sh}}$, measured in the Earth Centered Inertial frame (ECI). Figure 2 illustrates the geometry for shadow location calculations.

The shadow position vector $\mathbf{r}_{\mathrm{sh}}$ can be calculated at any particular time by defining the spacecraft position vector $\mathbf{r}_{s}$, and the Sun position vector $\mathbf{r}_{\text {sun. The magnitude of the }}$ shadow position vector is the Earth radius $R_{E}$

$$
\left\|\mathbf{r}_{\text {sh }}\right\|=R_{E}
$$

From Figure 2, the shadow angle $\theta_{\text {sh }}$ can be calculated as follows:

$$
\cos \theta_{\text {sh }}=\hat{r}_{s} \cdot \hat{r}_{\text {sun }}
$$




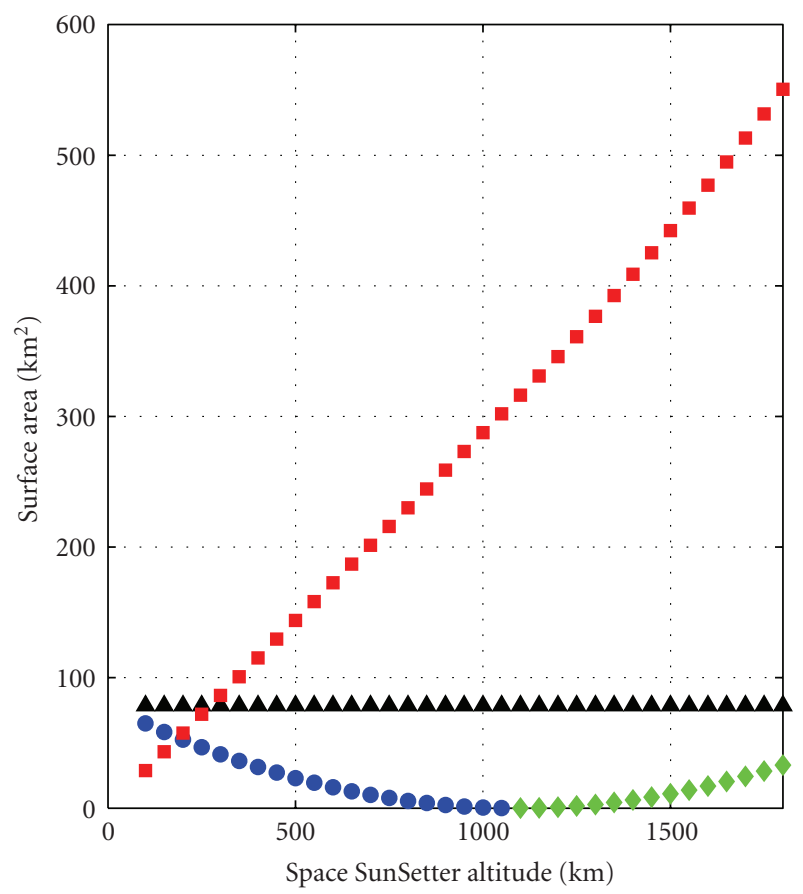

A Space SunSetter

- Complete shadow

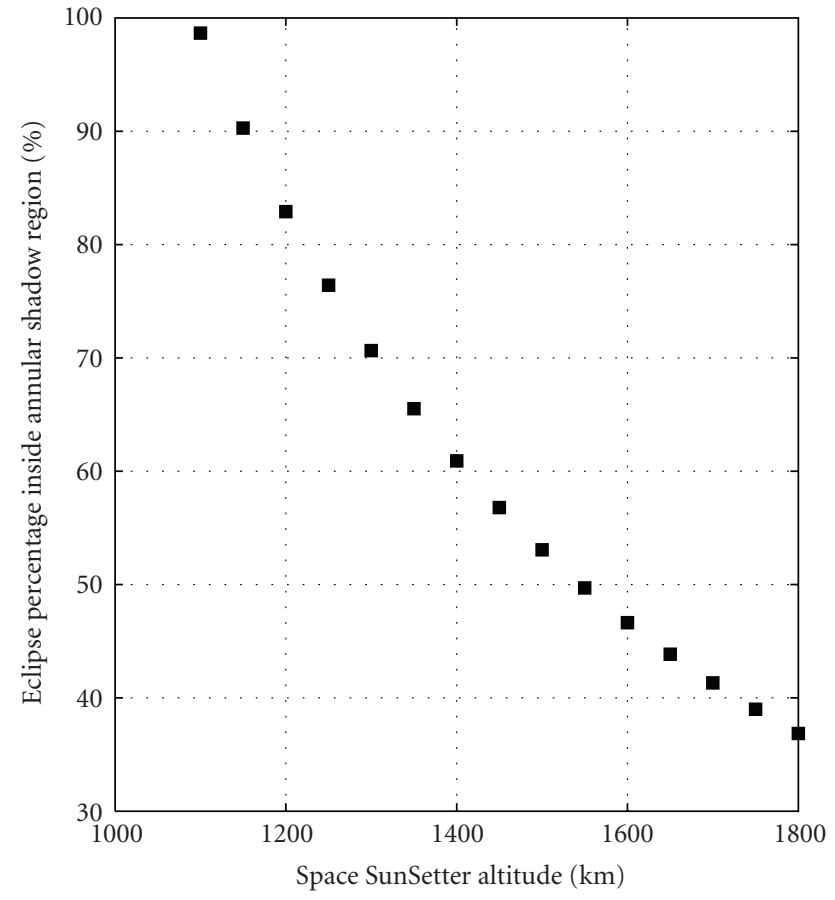

(b)

FIgURE 4: Space SunSetter with a radius of $5 \mathrm{~km}$ at $\theta_{\mathrm{sh}}=0$.
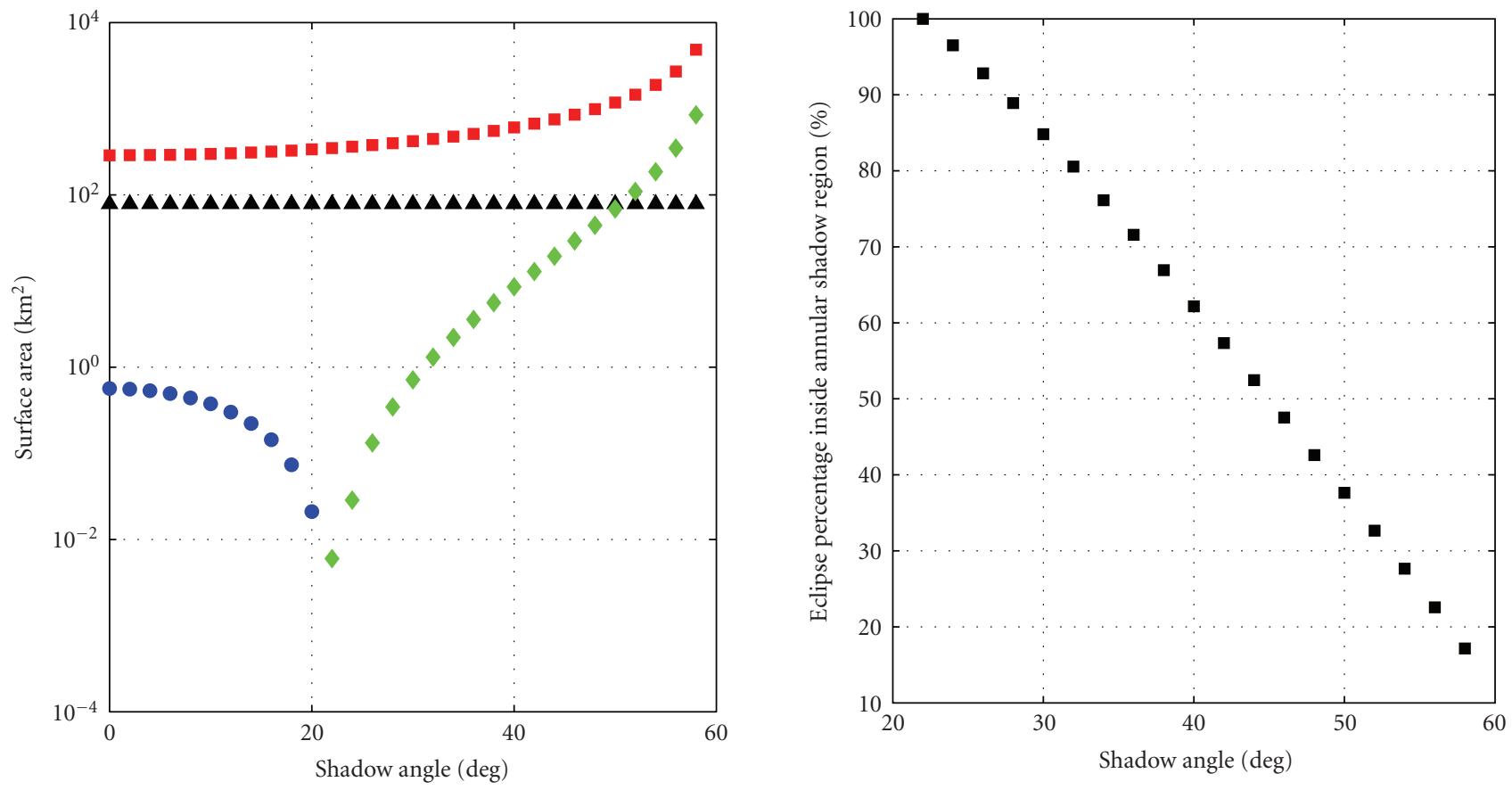

- Space SunSetter

- Complete shadow

(a)
- Partial shadow

- Annular shadow

(a) 


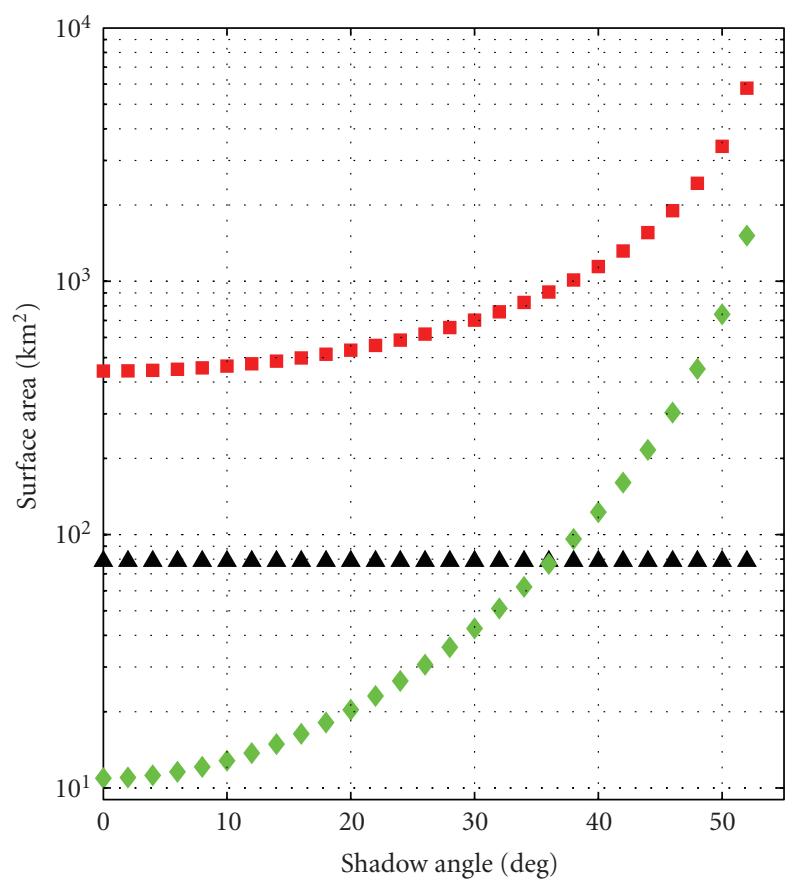

A Space SunSetter

- Annular shadow

- Partial shadow

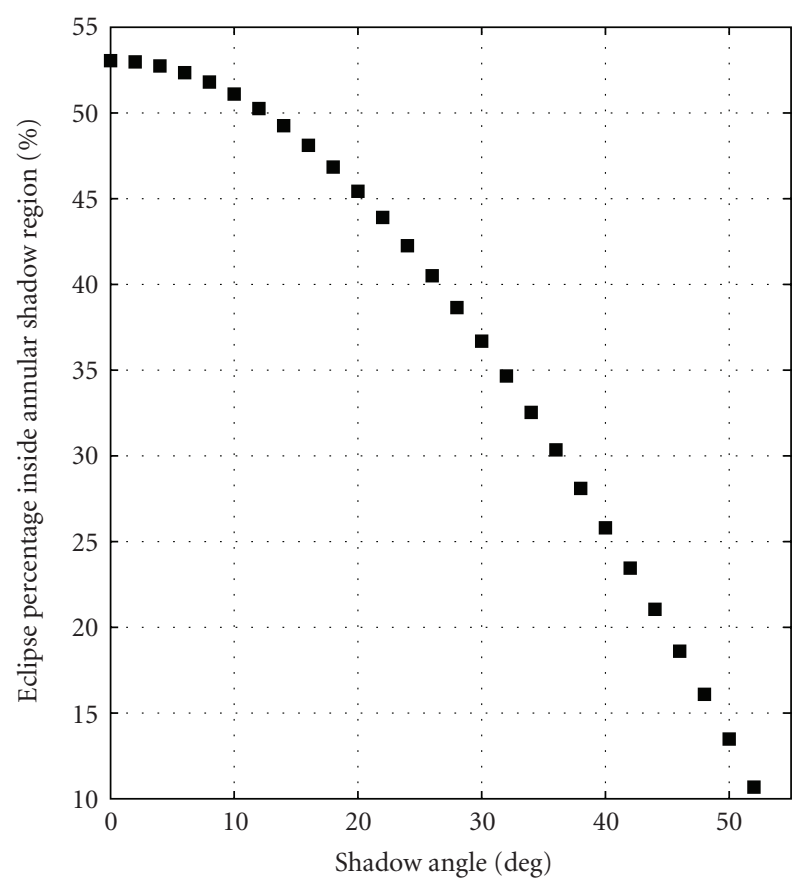

(b)

(a)

FIgURE 6: Space SunSetter with a radius of $5 \mathrm{~km}$ at $1500 \mathrm{~km}$ altitude.

where $\hat{r}_{s}$ and $\hat{r}_{\text {sun }}$ are the unit vectors in direction of spacecraft and the Sun, respectively. For the oblique triangle formed by $\mathbf{r}_{s}$ and $\mathbf{r}_{\mathrm{sh}}$ one has

$$
R_{E}^{2}=z^{2}+r_{s}^{2}-2 z r_{s} \cos \theta_{\text {sh }}
$$

Therefore,

$$
\begin{gathered}
z^{2}-\left(2 r_{s} \cos \theta_{\mathrm{sh}}\right) z+\left(r_{s}^{2}-R_{E}^{2}\right)=0, \\
z=r_{s} \cos \theta_{\mathrm{sh}} \pm \sqrt{R_{E}^{2}-r_{s}^{2} \sin ^{2} \theta_{\mathrm{sh}}}
\end{gathered}
$$

where $R_{E}$ is the Earth's radius, and $z$ is the distance between the spacecraft and its shadow point on the Earth's surface. The distance $z$ has two values; the smaller positive real value is chosen to calculate the shadow position vector:

$$
\mathbf{r}_{\mathrm{sh}}=\mathbf{r}_{s}-z \hat{r}_{\text {sun }}
$$

To get a shadow on the Earth's surface, the shadow angle should not exceed $\theta_{\mathrm{lim}}$, see Figure 7 . The angle $\theta_{\lim }$ is calculated as follows:

$$
\theta_{\lim }=\sin ^{-1}\left(\frac{R_{E}}{h+R_{E}}\right)
$$

where $h$ is the spacecraft altitude measured from the Earth surface, and $\theta_{\text {lim }}$ is in the first quadrant.

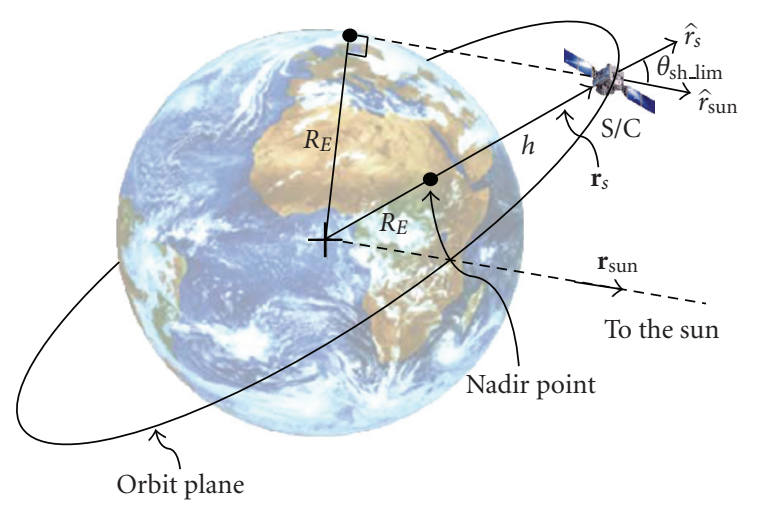

FIgURE 7: Shadow angle geometrical limitations.

3.2. Repeated-Shadow Sun-Synchronous Orbits. The shadow of a satellite in a repeated shadow track orbit repeats its track every certain period of time. If this period of time is an integer number of days, then the orbit may be called a repeated-shadow Sun-synchronous orbit. Therefore, after an integer number of successive shadow revolutions, and an integer number of days, the spacecraft shadow repeats its shadow track. A repeated-shadow Sun-synchronous orbit is favored for those missions where the shadow is required to revisit ground regions at the same daylight conditions. To derive the necessary conditions for a repeated-shadow 
Sun-synchronous orbit, we need to take into consideration the Earth rotation about its axis, the orbit parameters perturbations due to $J_{2}$ effect, and the Earth rotation around the Sun [19].

The Earth rotates through one revolution in its sidereal period of $\tau_{E}$, where $\tau_{E}=86164.1$ seconds. The effect of the Earth's rotation around its axis is a change in shadow point longitude $\Delta \varphi_{1}$ after one orbital revolution. Whereas there is no change in shadow point latitude, that is, $\Delta \lambda_{1}=0$. Figure 8 shows the effect of the Earth's rotation on shadow point track after one orbital nodal period. If the spacecraft's nodal period is $\tau$, then $\Delta \varphi_{1}$ may be written as [19]

$$
\Delta \varphi_{1}=-2 \pi \frac{\tau}{\tau_{E}} \mathrm{rad} / \text { orbit }
$$

The negative sign means a move towards the west [19]. Nodal period (node-to-node $\tau$ ) can be defined as [20],

$$
\tau=\frac{2 \pi}{n+\dot{M}_{o}+\dot{\omega}},
$$

where $n$ is the orbital mean motion, and the rates of change for the initial mean anomaly $M_{o}$ and the perigee argument $\omega$ due to $J_{2}$ perturbation have the expressions

$$
\begin{aligned}
& \dot{M}_{o}=\frac{3 n J_{2}\left[3 \cos ^{2} i-1\right]}{4\left(1-e^{2}\right)^{3 / 2}}\left(\frac{R_{E}}{a}\right)^{2}, \\
& \dot{\omega}=-\frac{3 n J_{2}\left[1-5 \cos ^{2} i\right]}{4\left(1-e^{2}\right)^{2}}\left(\frac{R_{E}}{a}\right)^{2},
\end{aligned}
$$

respectively. Equations (9) are derived from geopotential perturbation theory [18] by considering $J_{2}$ perturbations, only.

The second effect on RSTO is the orbit perturbations [19]. The first zonal harmonic, $J_{2}$, which represents the Earth's oblateness, will be assumed the only perturbing force that affects the spacecraft. $J_{2}$ perturbation causes secular motion in three orbital elements: ascending node $\Omega$, argument of perigee $\omega$, and mean anomaly $M$. The average changes of the remaining orbital elements, semimajor axis $a$, eccentricity $e$, inclination $i$, per orbit can be considered to be zero. Figure 9 illustrates the effect of $J_{2}$ perturbation on the shadow track.

After one spacecraft orbital period $T$, and due to only $J_{2}$ perturbation, the shadow track will be changed by $\Delta \lambda_{2}$ in latitude and by $\Delta \varphi_{2}$ in longitude, where $\Delta \varphi_{2}=\Delta \varphi_{21}+\Delta \varphi_{22}$. The regression of the line of nodes $\dot{\Omega}$ contributes only $\Delta \varphi_{22}$, while $\Delta \lambda_{2}$ and $\Delta \varphi_{21}$ are the direct effect of average change in argument of perigee $\dot{\omega}$ and mean anomaly $\dot{M}$. However, after one spacecraft nodal period $\tau$, the effect of $\dot{\omega}$ and $\dot{M}$ disappears in shadow track calculations. Therefore, $\Delta \lambda_{2}$ and $\Delta \varphi_{21}$ are equal to zero. Hence, the orbit perturbations contribute [19]

$$
\Delta \varphi_{2}=\Delta \varphi_{22}=-\frac{3 \pi J_{2} R_{E}^{2} \cos i}{a^{2}\left(1-e^{2}\right)^{2}} \mathrm{rad} / \text { orbit. }
$$

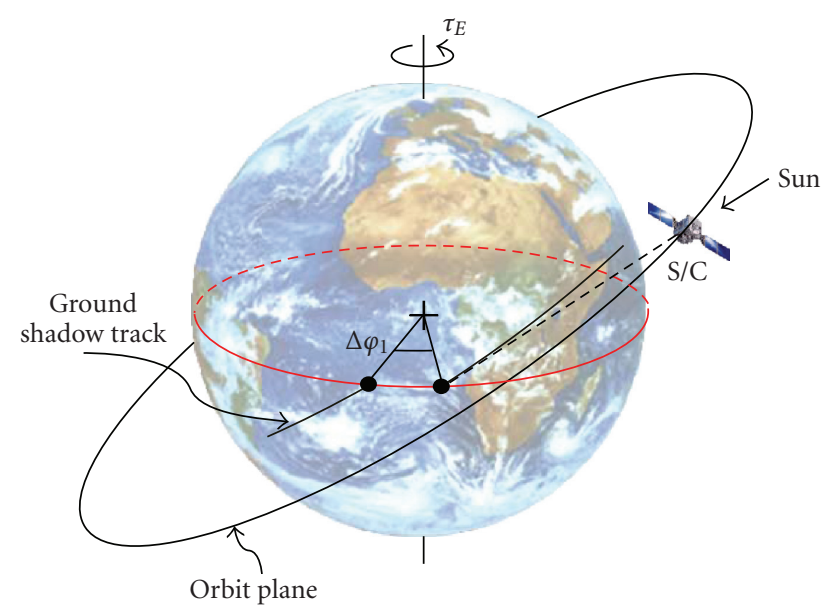

FIGURE 8: The effect of the Earth's rotation around its axis on shadow track.

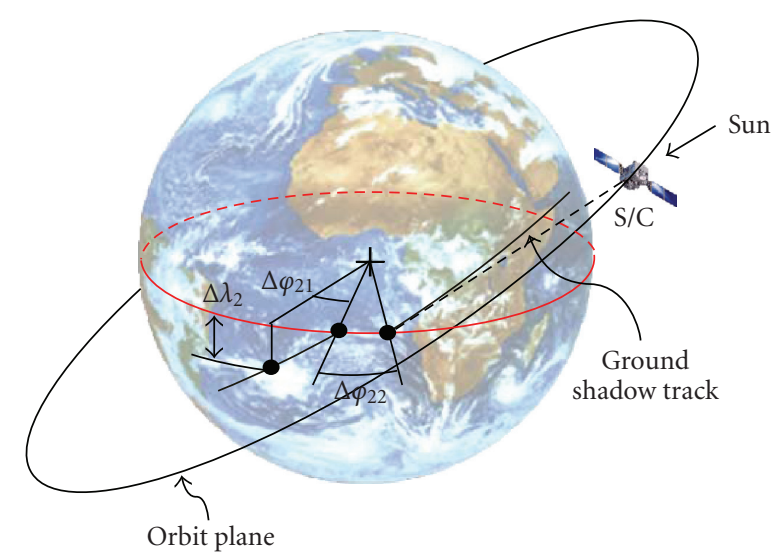

FIGURE 9: The effect of $J_{2}$ perturbation on the ground shadow track.

In case of selecting the repeated shadow track orbit, as well as a Sun-synchronous orbit, the required rotation rate is [19]

$$
\Delta \varphi_{2}=2 \pi \frac{\tau}{\tau_{\mathrm{ES}}} \mathrm{rad} / \text { orbit }
$$

where $\tau_{\mathrm{ES}}=3.155817 \times 10^{7}$ seconds is the orbital period of the Earth round the Sun.

The third effect on RSTO is the Earth's rotation around the Sun [18]. Figure 10 shows the change in shadow track due to the Earth's rotation around the Sun. The Earth completes one revolution around the Sun once every year. Therefore, the Sun's rotation rate, $\Delta \theta_{\text {sun }}$, for a spacecraft with nodal period $\tau$ is [18]

$$
\Delta \theta_{\text {sun }}=2 \pi \frac{\tau}{\tau_{\mathrm{ES}}} \mathrm{rad} / \text { orbit. }
$$

The Earth rotates around the Sun in the ecliptic plane while Earth's equatorial plane is inclined about $23.5^{\circ}$ to the ecliptic. This phenomenon explains the change in both latitude $\Delta \lambda_{3}$ and longitude $\Delta \varphi_{3}$ of a spacecraft's shadow point on earth surface after one nodal period as shown in Figure 10. 


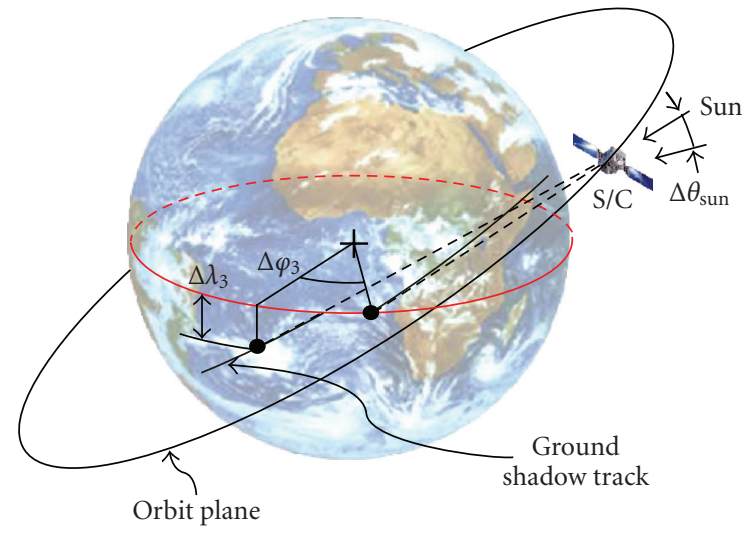

Figure 10: The effect of Earth rotation around the Sun on the ground shadow track.

The total changes in shadow point latitude $\Delta \lambda_{\text {nodal }}$ and longitude $\Delta \varphi_{\text {nodal }}$ after one spacecraft nodal period $\tau$ are

$$
\begin{gathered}
\Delta \varphi_{\text {nodal }}=\Delta \varphi_{1 \text { nodal }}+\Delta \varphi_{2 \text { nodal }}+\Delta \varphi_{3 \text { nodal }} \mathrm{rad} / \text { orbit } \\
\Delta \lambda_{\text {nodal }}=\Delta \lambda_{3 \text { nodal }} \mathrm{rad} / \text { orbit. }
\end{gathered}
$$

To select a repeated shadow track orbit, the shadow track should revisit the same ground locations, latitude and longitude, after an integer number of shadow revolutions within a certain period of time (integer number of days for Sun-synchronous orbits). Thus, it is required that some integral number of orbits later the accumulated value of the total change in shadow longitude will equal $2 \pi$, and the total change in shadow latitude will equal zero [19]. Therefore, the total change in shadow longitude $\sum_{i=1}^{n}\left|\Delta \varphi_{\text {shadow }}\right|$ and the total change in shadow latitude $\sum_{i=1}^{n}\left|\Delta \lambda_{\text {shadow }}\right|$ can be formulated as follows:

$$
\begin{gathered}
\sum_{i=1}^{n}\left|\Delta \varphi_{\text {shadow }}\right|=2 \pi m, \\
\sum_{i=1}^{n}\left|\Delta \lambda_{\text {shadow }}\right|=0,
\end{gathered}
$$

where $n$ is the total number of successive shadow revolutions performed and $m$ is the number of Earth revolutions (equivalent to days) before an identical shadow track occurs.

After completing one shadow revolution, the shadow track changes only by $\Delta \varphi_{\text {sh }}$ in longitude, while the change in shadow latitude $\Delta \lambda_{\text {sh }}$ is equal to zero. The period needed to complete one shadow revolution, by keeping $\Delta \lambda_{\text {sh }}=0$, is the shadow nodal period $\tau_{\text {sh }}$. It can be calculated from the following formula:

$$
\tau_{\mathrm{sh}}=\frac{2 \pi}{\omega_{\mathrm{sh}}}
$$

where $\omega_{\text {sh }}$ is the rate of change of ground shadow point position measured in the shadow track on Earth surface. $\omega_{\text {sh }}$ depends on two main parameters. The first is the rate of change of the spacecraft position along the ground shadow track orbit. The second is the rate of change of the EarthSun vector in the same orbit. Figure 11 shows latitude and longitude changes for both shadow and spacecraft ground track after shadow nodal period $\tau_{\text {sh }}$ and spacecraft nodal period $\tau$. Shadow point changes in both latitude and longitude with different rate from the subspacecraft point "nadir point." This phenomenon is a direct result of variation of Earth-Sun vector with time, as explained in Figure 10.

A numerical approach that is implemented to calculate the shadow nodal period $\tau_{\mathrm{sh}}$ of a spacecraft has a nodal period $\tau$. As illustrated in Figure 11, the change in shadow latitude of a spacecraft propagated for $\tau_{\mathrm{sh}}$ is equal zero. Hence, propagating a spacecraft for any other period results in a nonzero change in shadow point latitude. This fact is used to calculate an accurate value for a spacecraft shadow nodal period at a certain day time. The following formula is used to get an accurate value for $\tau_{\mathrm{sh}}$ :

$$
T=T\left[1-\frac{\Delta \lambda_{\mathrm{sh}}}{2 \pi}\right]
$$

First, the change in shadow latitude $\Delta \lambda_{\text {sh }}$ is calculated after propagating the spacecraft for a nodal period $\tau$. Then, the spacecraft nodal period is substituted in the righthand side of (16) with its corresponding change in shadow latitude. The calculated value of $T$ in the left-hand side can be considered as an initial value of shadow nodal period $\tau_{\text {sh }}$. This initial value is used to propagate the spacecraft and calculate its corresponding $\Delta \lambda_{\text {sh }}$. Once again, (16) is used to generate a correlated value of the shadow nodal period. Finally, an accurate value of $\tau_{\text {sh }}$ can be calculated after a limited number of iterations. At this point, the total change in shadow location after $\tau_{\mathrm{sh}}$ is in shadow longitude $\Delta \varphi_{\mathrm{sh}}$, while $\Delta \lambda_{\text {sh }}$ equals zero.

From the previous discussion, we realize that the shadow nodal period $\tau_{\text {sh }}$ depends on the orbital elements and on the Sun's position, which is a function of time. It can be concluded then that the shadow nodal period of a spacecraft orbiting the Earth is also a function of time. Hence, the change in shadow point longitude after propagating the spacecraft for $\tau_{\text {sh }}$ is also a function of time.

\section{Optimization}

For the purpose of designing an RSTO that has its shadow repeats over a certain ground site with a maximum duration for the shadow per day over this site and a minimum number of revolutions per day, an optimization problem is formulated. The parameters to be optimized are the spacecraft orbital elements. These parameters are subjected to constraints to provide the repeated shadow track conditions. The fitness $F_{i}$ (the cost function to be maximized) at a design point is defined as follows:

$$
F_{i}=f_{1} J_{i 1}+f_{2} J_{i 2}
$$




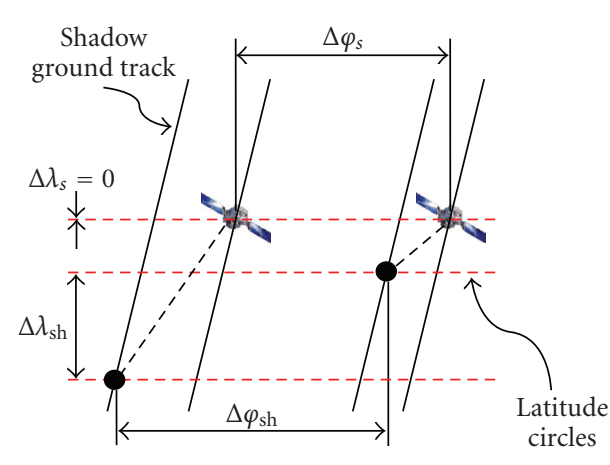

(a)

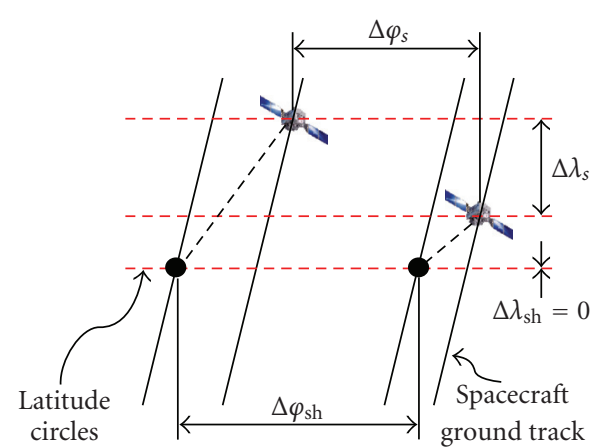

(b)

Figure 11: Latitude and longitude changes for both shadow and spacecraft ground track after: (a) spacecraft nodal period $\tau$, (b) shadow nodal period $\tau_{\text {sh. }}$.

where $f_{1}$ and $f_{2}$ are the fitness weight parameters, and $J_{i 1}$ and $J_{i 2}$ are the fitness terms for the $i$ th design point which can be calculated as follow:

$$
\begin{aligned}
J_{i 1} & =\frac{T_{\text {tsh }}}{m}, \\
J_{i 2} & =-\frac{n}{m},
\end{aligned}
$$

where $T_{\text {tsh }}$ is the target shadow period over a specific site, $n$ is the number of successive shadow revolutions, and $m$ is the number of days before an identical shadow track will occur.

The values of the fitness weight parameters $f_{1}$ and $f_{2}$ are chosen to maximize the effect of $J_{i 1}$. The negative sign in $J_{i 2}$ indicates that this term has to be minimized. The previous expression is valid only for the design points which provide the repeated shadow track conditions. Otherwise, the fitness $F_{i}$ for any nonRSTO design point is chosen to be a small negative value less than the minimum value of the fitness function for any RSTO design point. To see how the fitness function $F_{i}$ changes with the change in the spacecraft orbital elements (optimization parameters), $F_{i}$ is plotted versus each couple of orbital elements in Figure 12.

The objective function shown in Figure 12 is a multi minima function. Classical optimization methods fail to find the optimal solution in this case. On the other hand, genetic algorithms can be implemented to find a highly fit solution for the problem. Genetic algorithms are search algorithms based on the mechanics of nature selection and natural genetics. They combine survival of the fittest among string structures with a structured yet randomized information exchange to form a search algorithm [12].

In genetic algorithms, a design point is called a member. A finite number of members are randomly generated to create the initial population. Generations of this population are created using genetic algorithms operations such as reproduction, crossover, and mutation. Each generation is a single iteration. At each generation, members that are fittest are selected in a parent's pool. The fitness of a member is determined according to the objective, the cost function in this case. These parents are then used to create the new

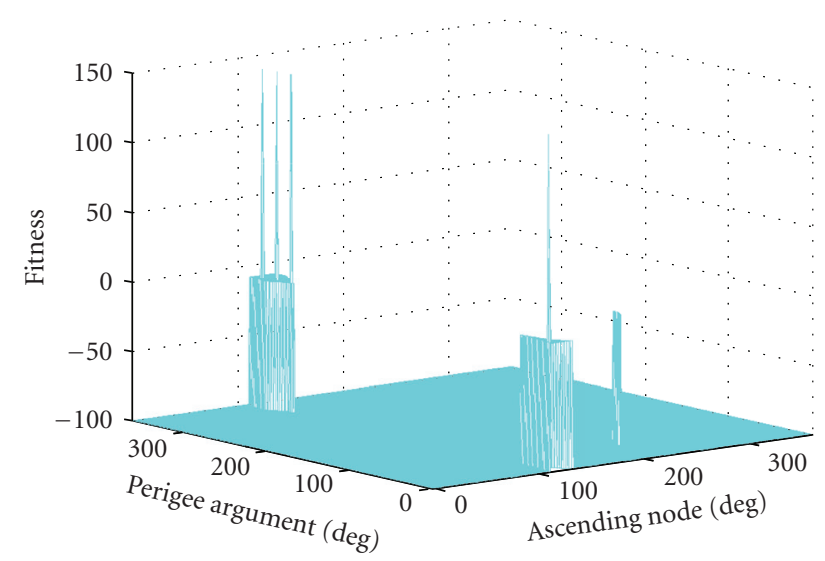

FIgURE 12: The fitness function variation versus the orbital elements.

generation. This process leads to the evolution of populations of individuals that are fittest.

Each member, design point, in the population represents six design variables. Five are orbital elements that are coded as continuous design variables. These variables are eccentricity $e$, inclination $i$, right ascension of ascending node $\Omega$, argument of perigee $\omega$, and true anomaly $\vartheta$. The desired number of days before an identical shadow track will occur, $m$, is coded as a discrete design variable. Each member is presented in a binary format as a binary string. This string contains the binary representation of all the design variables values at the corresponding design point. The number of bits for each variable determines its accuracy. Discrete variable would be assigned a unique binary string. The number of bits $q_{m}$ for the discrete design variable, $m$, depends on the upper bounds of the desired design variable, $m_{\max } \cdot q_{m}$ for the discrete design variable $m$ is determined as follows:

$$
q_{m}=1 \text { bit, } \quad \text { if } m_{\max }=1 \text { day, }
$$

or,

$$
2^{q_{m}} \leq m_{\max }, \quad \text { if } m_{\max }>1 \text { day. }
$$


TABLE 1: The values of the lower and upper bounds, accuracy, and number of bits for each continuous design variable.

\begin{tabular}{lccc}
\hline Continuous design variable & Lower bounds & Upper bounds & \multicolumn{2}{c}{ Accuracy } & $A_{i c}$ \\
$A_{i}$ & $A_{i \min }$ & $A_{i \max }$ & 0.001 \\
\hline Eccentricity $e$ & 0 & 0.6 & 0.001 \\
Inclination $i(\mathrm{deg})$ & 90 & 180 & 0.001 \\
Ascending node $\Omega(\mathrm{deg})$ & 0 & 360 & 0.001 \\
Perigee argument $\omega(\mathrm{deg})$ & 0 & 360 & 12 \\
True anomaly $9(\mathrm{deg})$ & 0 & 360 & 0.001 \\
\hline
\end{tabular}

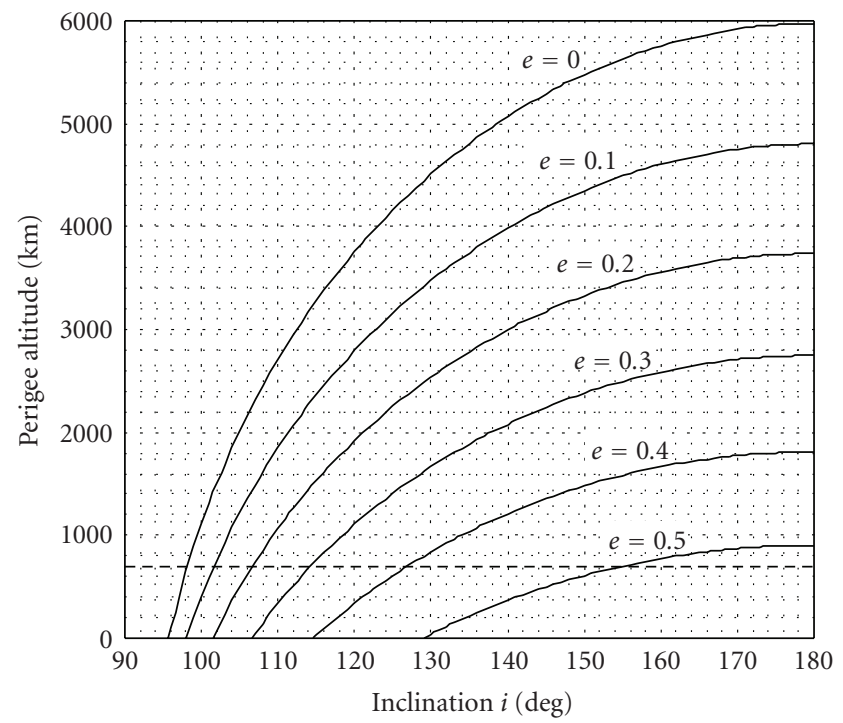

Figure 13: The orbit parameters limitations for a Sun-synchronous orbit.

For the continuous design variable, the number of bits $q_{i}$ for a continuous design variable $A_{i}$ is selected according to the following inequality:

$$
2^{q_{i}} \leq \frac{A_{i \max }-A_{i \min }}{A_{i c}}+1
$$

where $A_{i \min }$ and $A_{i \max }$ are the lower and upper bounds on the $i$ th continuous design variable, and $A_{i c}$ is the desired accuracy. Table 1 shows the values of the lower and upper bounds, accuracy, and number of bits for each continuous design variable. For a Sun-synchronous orbit, the orbit inclination range is limited as shown in Figure 13. The inclination should be more than $90^{\circ}$ to provide the desired regression of node of a Sun-synchronous orbit. Also, a limitation on the eccentricity to be less than 0.6 is applied to guarantee certain minimum altitude for the spacecraft.

The strings for all members in the initial population are selected randomly within the design variables limitations. Each member turned out to be the five orbital elements plus the desired number of days before an identical shadow track will occur. These values are used to evaluate the fitness function $F_{i}$ for each design point separately according to the following steps. First, the semimajor axis is calculated to provide the condition of a Sun-synchronous orbit. The perigee altitude is determined to check the feasibility of the orbit according to Figure 13. The semimajor axis $a$ and the perigee altitude $h_{\text {perigee }}$ can be calculated for each design point as follows [18]:

$$
\begin{gathered}
a=\left[-\frac{3 R_{E}^{2} J_{2} \sqrt{\mu}}{2\left(1-e^{2}\right)^{2} \dot{\Omega}} \cos i\right]^{2 / 7}, \\
h_{\text {perigee }}=a(1-e)-R_{E},
\end{gathered}
$$

where $\dot{\Omega}=1.991 \times 10^{-7} \mathrm{rad} / \mathrm{sec}$ is the desired rate of change of the orbital ascending node to provide a Sun-synchronous orbit.

Now, the spacecraft location for each design point is known from the 6 orbital elements. Hence, shadow calculations, discussed in Section 3.1, are used to decide whether that design point has a shadow on the Earth's surface or not. For any nonshadow members, the fitness is chosen to be a small negative value. The ground shadow location is determined for those members satisfying ground shadow at a predefined initial time. Therefore, the shadow nodal period $\tau_{\text {sh }}$ can be calculated according to the numerical technique described in Section 3.2. Then, the shadow track is propagated by $\tau_{\text {sh }}$ over a limited number of revolutions $n$ until the total change in shadow longitude $\sum_{i=1}^{n}\left|\Delta \varphi_{\text {shadow }}\right|$ accumulates $2 \pi m$ or more. Using $\tau_{\text {sh }}$ in shadow propagation guarantees total change in shadow latitude $\sum_{i=1}^{n}\left|\Delta \lambda_{\text {shadow }}\right|$ equal to zero. The total number of shadow revolutions can be considered as the integer number of orbits required for shadow repetition in $m$ days. The summation of shadow nodal period $\sum_{i=1}^{n} \tau_{\mathrm{sh}}$ is determined, which is equal to or greater than $m$ days.

Not all design points in the population satisfy the repeated shadow track conditions. Some members do not have a shadow track on Earth surface. The rest of the design points, which provide ground shadow track, have $\sum_{i=1}^{n}\left|\Delta \varphi_{\text {shadow }}\right|>2 \pi m$, or $\sum_{i=1}^{n} \tau_{\text {sh }}>m$. In this case, an equivalent design point is calculated to check whether this orbit could be modified to an RSTO or not. This is orbital elements tuning. It is conducted by changing (tuning) only one orbital element to satisfy the repeated shadow track conditions. The inclination is selected to be the tuning element. Changing the inclination value leads to a change in the semimajor axis to keep the Sun-synchronous orbit condition.

The inclination is tuned to guarantee that the total change in shadow longitude $\sum_{i=1}^{n}\left|\Delta \varphi_{\text {shadow }}\right|$ accumulates 


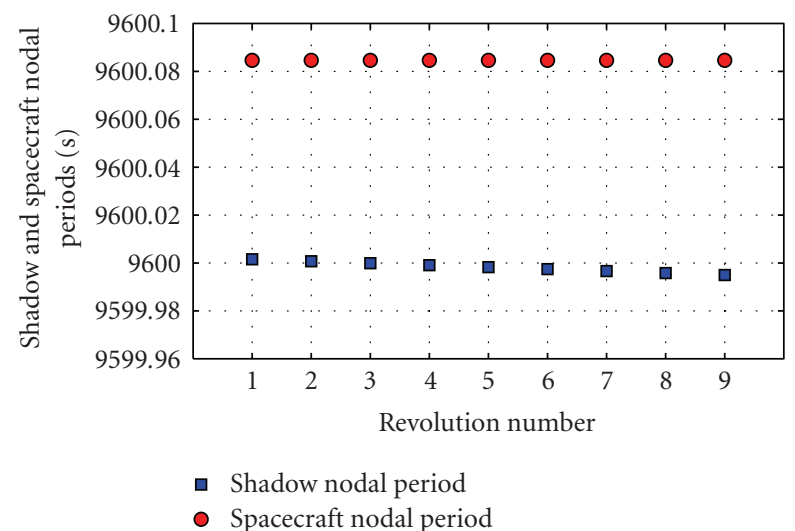

Figure 14: The difference between the shadow nodal period and spacecraft nodal period.

$2 \pi m$ within an acceptable error tolerance. The orbital parameters tuning is not enough to decide that the equivalent design point represents an RSTO. The summation of shadow nodal period $\sum_{i=1}^{n} \tau_{\text {sh }}$ for the modified design point must be equal to the integer number $m$, satisfying these conditions guarantees that the design point in the population corresponds to a repeated shadow track orbit.

The target shadow period $T_{\text {tsh }}$ is required to calculate the fitness of the RSTOs design points. To calculate $T_{\text {tsh }}$, the spacecraft's shadow is propagated for the whole $m$ days required for shadow repetition. Then, the target shadow period is measured as the total time of shadow existence over a target site in $m$ days. To get an accurate value of $T_{\text {tsh }}$, the orbit propagation should be performed using a small time step, about 1 second Given the problem in question, this can be quite time consuming. To minimize the run time, a varying time step is used during shadow propagation. For the shadow points which are close enough to the target site, a small time step is used in propagation. When the shadow is not a concern, a larger time step is used.

Finally, by calculating $T_{\text {tsh }}, m$, and $n$, the fitness $F_{i}$ can be determined for each design point. The fittest members in the initial population are selected as parents for the next generation. Genetic algorithms operations are then used to generate a new generation which contains better design points. This process is repeated for a predefined number of generations. Finally, the fittest members in the last generation are selected. The most fit design point is the member which represents a repeated shadow track orbit passes over a particular target site with minimum number of revolutions and maximum target shadow period at a given time.

\section{Numerical Results}

A numerical example for an RSTO is first presented, then the results of the developed genetic algorithm tool are presented for maximum shadow duration over a certain ground site.

To provide a numerical example for an RSTO, the orbital elements are selected arbitrary such that the Sunsynchronous orbit condition is satisfied and such that the satellite has a shadow on Earth surface, initially. Tuning for the orbital elements is then done to calculate the exact elements that satisfy the RSTOs conditions. The inclination and the semimajor axis are the two orbital elements that are modified in tuning for the RTSO.

A set of orbital elements, arbitrarily selected, are considered as an initial guess for an RSTO, on the first of July 2008. The required number of days, before the shadow track repeats, is $m=1$ day. The values of the initial orbital elements ( $i, e, \Omega, \omega$, and $\vartheta$ ), are selected such as to satisfy the Sun-synchronous orbit condition and to have a shadow on earth surface at the given initial date. Table 2 shows the initial and the tuned orbital elements and the shadow and spacecraft track characteristics for each set of orbital elements.

The tuned orbital elements represent an RSTO. Tuning the inclination, from $120^{\circ}$ to $116.017^{\circ}$, results in decreasing the semimajor axis, from $10133.879 \mathrm{~km}$ to $9761.798 \mathrm{~km}$, to maintain a Sun-synchronous orbit. Both the initial and the tuned orbits provide a very small value, almost zero, for the total change in shadow latitude after $n=9$ shadow nodal periods, $\tau_{\text {sh }}$. On the other hand, the total change in spacecraft latitude, in both cases, is much higher than the total change in shadow latitude, that is, $\sum_{i=1}^{n}\left|\Delta \lambda_{s / c}\right| \gg \sum_{i=1}^{n}\left|\Delta \lambda_{\text {shadow }}\right|$.

The characteristics of the RSTO can be obtained from the numerical results. The total change in shadow longitude and the summation of shadow nodal period after completing nine shadow revolutions are approximately $360^{\circ}$ and 86400 seconds, respectively. These values are equivalent to one Earth revolution around its axis, $m=1$ day, within acceptable numerical error margins. It is also concluded from the numerical results that the shadow nodal period for an RSTO is not constant with time. Figure 14 shows the difference between the shadow nodal period and the spacecraft nodal period, which is constant with time. The total change in longitude and latitude of spacecraft ground track is not equal to the total change in shadow longitude and latitude. This explains the concept of shadow repetition illustrated in Figure 11. Figure 15 explains the variation in longitude and latitude for both shadow and spacecraft ground track of the RSTO. Figure 16 shows the spacecraft ground track and the repeated shadow track for the RSTO. Both of the tracks are propagated for two days, $m+1$, to demonstrate the concept of shadow repetition.

Genetic algorithms are used to select the best RSTO appropriate for a specific ground site at a certain date. The most fit orbital elements should provide a shadow repetition with a minimum number of shadow revolutions during an integer number of days and a maximum shadow duration over the given ground site. A numerical example is performed to generate the best orbital elements of an RSTO starting at the first day of July 2008. The ground site has longitude and latitude of $51.3484^{\circ}$ and $25.1528^{\circ}$, respectively. The site shadow duration $T_{\text {tsh }}$ is calculated based on the assumption that the site size is $10 \mathrm{~km}$ radius from the site center. The shadow duration is the time during which the shadow is within this radius.

The population size is selected to be 100, and the number of generation is 200 . The probability of crossover is considered 0.9 , while the mutation probability is taken between 0.01 and 0.08 . $10 \%$ of the most fit members 


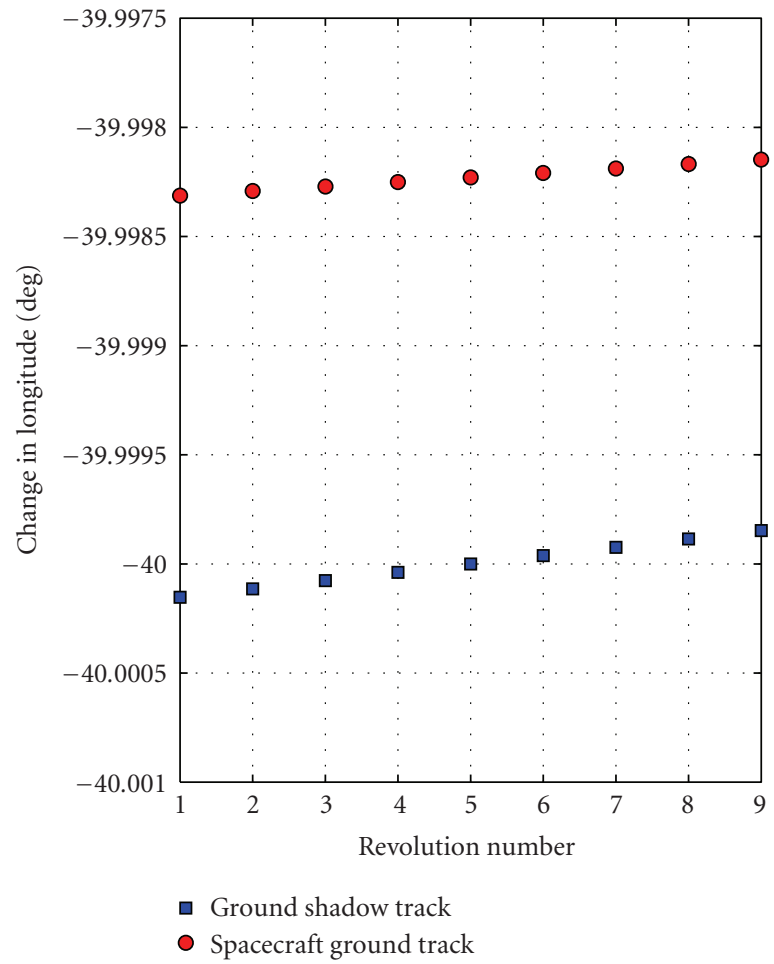

(a)

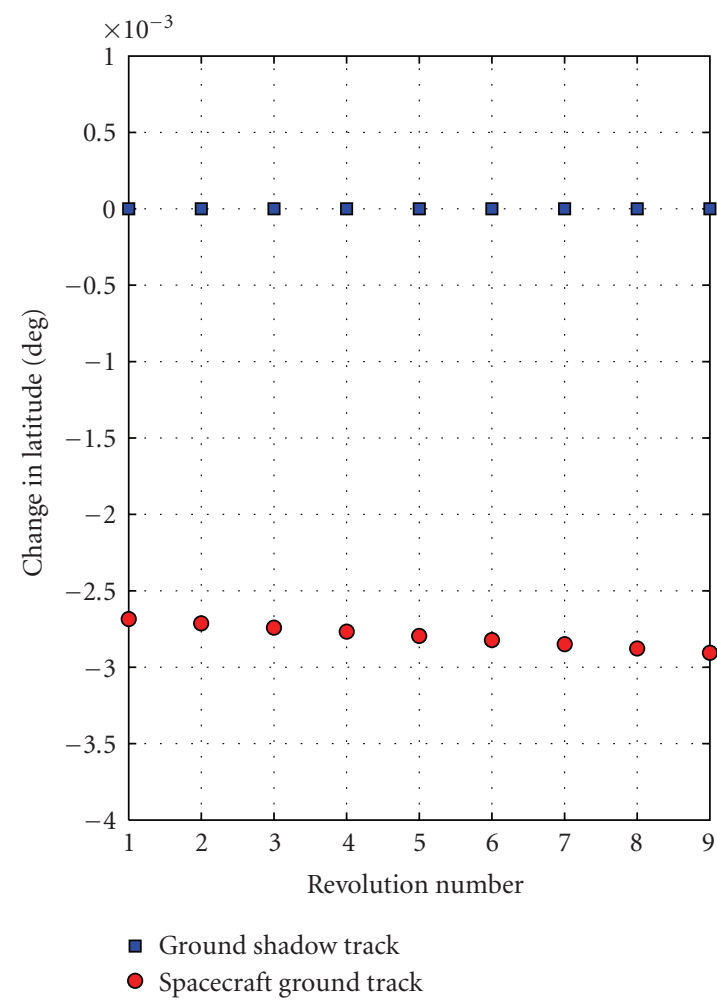

(b)

FIGURE 15: The variation in longitude and latitude for both shadow and spacecraft ground track of an RSTO.

TABLE 2: The initial and modified orbital elements and their shadow and spacecraft track characteristics.

\begin{tabular}{|c|c|c|}
\hline $\begin{array}{l}\text { Orbital parameters and shadow and spacecraft track } \\
\text { characteristics }\end{array}$ & Initial orbit & Modified orbit (RSTO) \\
\hline Inclination, $i$ (deg) & 120 & 116.017 \\
\hline Eccentricity, $e$ & 0.01 & 0.01 \\
\hline Ascending node, $\Omega$ (deg) & 120 & 120 \\
\hline Perigee argument, $\omega(\mathrm{deg})$ & 30 & 30 \\
\hline True anomaly, $\vartheta(\mathrm{deg})$ & 0 & 0 \\
\hline Semi major axes, $a(\mathrm{~km})$ & 10133.879 & 9761.798 \\
\hline No. of shadow revolution, $n$ & 9 & 9 \\
\hline \multicolumn{3}{|l|}{ Total change in shadow longitude, } \\
\hline$\sum_{i=1}^{n}\left|\Delta \varphi_{\text {shadow }}\right|(\operatorname{deg})$ & -380.713 & -360 \\
\hline \multicolumn{3}{|l|}{ Total change in shadow latitude, } \\
\hline$\sum_{i=1}^{n}\left|\Delta \lambda_{\text {shadow }}\right|(\operatorname{deg})$ & $1.930 \times 10^{-10}$ & $2.916 \times 10^{-10}$ \\
\hline \multicolumn{3}{|l|}{ Summation of shadow nodal period, } \\
\hline$\sum_{i=1}^{n} \tau_{\mathrm{sh}}(\mathrm{sec})$ & 91372.036 & 86399.985 \\
\hline \multicolumn{3}{|l|}{ Total change in spacecraft longitude, } \\
\hline$\sum_{i=1}^{n}\left|\Delta \varphi_{s / c}\right|(\operatorname{deg})$ & -380.696 & -359.984 \\
\hline \multicolumn{3}{|l|}{ Total change in spacecraft latitude, } \\
\hline$\sum_{i=1}^{n}\left|\Delta \lambda_{s / c}\right|(\operatorname{deg})$ & -0.0282 & -0.0251 \\
\hline
\end{tabular}




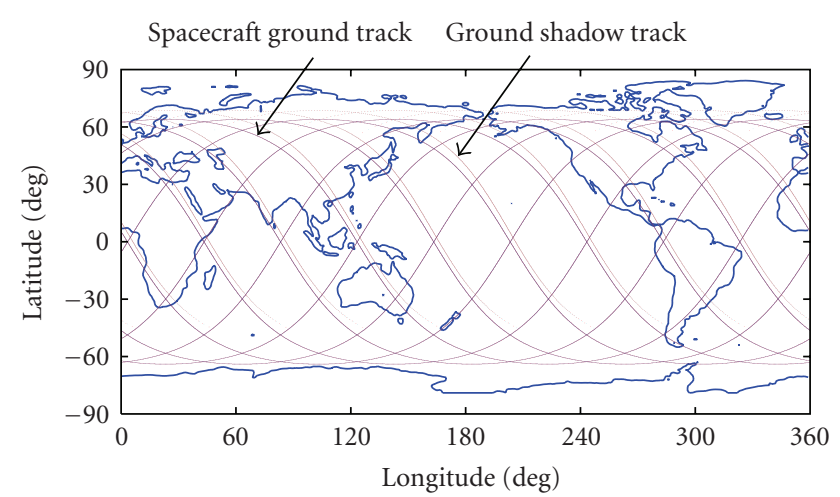

FIGURE 16: The spacecraft ground track and the ground shadow track for an RSTO.

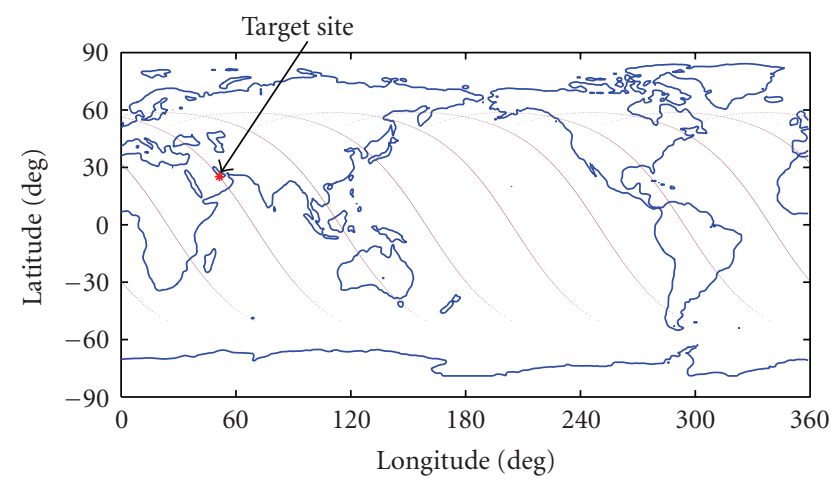

FIGURE 17: The ground shadow track for the repeated shadow track orbit obtained by genetic algorithm.

in each generation are copied automatically into the next generation without performing any genetic operations. The values of the weight parameters $f_{1}$ and $f_{2}$ are selected to be 2 and 1 , respectively. The fittest orbital obtained by genetic algorithms represents an RSTO that passes over the given site. This orbit completes 8 successive shadow revolutions in one day to provide shadow repetition over the desired site. The orbital elements of the obtained solution are eccentricity 0.2505 , semimajor axis $10560.2095 \mathrm{~km}$, inclination $120.4955^{\circ}$, argument of perigee $0.0879^{\circ}$, ascending node $113.2609^{\circ}$, and true anomaly $22.8544^{\circ}$. The total shadow duration over the Earth surface is 14560 seconds per day. While the site shadow duration is six seconds per day. Figure 17 shows the ground shadow track for the RSTO obtained by genetic algorithm.

\section{Conclusion}

In this paper, the concept of repeated shadow track orbit is introduced. Constraints on the orbital elements are derived to obtain a repeated shadow track orbit. For a two-body motion perturbed only by the $J_{2}$, the orbital elements need to be updated continuously to maintain the repetition of the shadow track on ground. An optimization tool has been developed using a genetic algorithm approach to obtain the orbit with maximum time duration over a given ground site. Results show that for a natural orbit, that is, without a control, the maximum duration time for a shadow over a point on ground will be in the order of few seconds in one orbit revolution.

\section{References}

[1] http://www.nas.nasa.gov/Services/Education/SpaceSettlement/ CoEvolutionBook/EDUC.HTML.

[2] "Space-based solar power as an opportunity for strategic security, phase 0 architecture feasibility study," Report to the Director, National Security Space Office, Interim Assessment, 2007.

[3] R. Kunzig, Geoengineering: How to Cool Earth-At a Price, Scientific American, 2008.

[4] Office of Technology Assessment, "Solar power satellites," Tech. Rep. 81-600129, NTIS order no. PB82-108846, Library of Congress Catalog Card, 1981.

[5] Committee for the Assessment of NASA's Space Solar, Power Investment Strategy, Aeronautics and Space Engineering Board, and National Research Council, Laying the Foundation for Space Solar Power: An Assessment of NASA's Space Solar Power Investment Strategy, National Academy Press, Washington, DC, USA, 2001.

[6] G. Landis, "Reinventing the solar power satellite, NASA/ TM2004-212743, IAC02R.1.07," in Proceedings of the 53rd International Astronautical Congress, Houston, Tex, USA, October 2002.

[7] L. Summerer and F. Ongaro, "Advanced space technology for 21st century energy systems: solar power from space," in Proceedings of the 2nd International Conference on Recent Advances in Space Technologies (RAST '05), pp. 16-23, Istanbul, Turkey, June 2005.

[8] V. Blandow, P. Schmidt, W. Weindorf, et al., "Earth and space based power generation systems-a fair comparison for long-term sustainable energy supply," GSP Final Report 17682/03/NL/EC, European Space Agency, 2005.

[9] Communication with Mr. Omer, of the Advanced Technical Solutions Gmbh, Gmbh, Berlin, Germany.

[10] B. Edwards, “The space elevator, NIAC phase I," Tech. Rep., Eureka Scientific, 2002, http://www.niac.usra.edu/studies/ 472Edwards.html.

[11] B. Edwards, "The space elevator, NIAC phase II," Tech. Rep., Eureka Scientific, 2002, http://www.niac.usra.edu/studies/ 521Edwards.html.

[12] D. Goldberg, Genetic Algorithms in Search, Optimization, and Machine Learning, Addison-Wesley, New York, NY, USA, 1989.

[13] Y. Kim and D. Spencer, "Optimal spacecraft rendezvous using genetic algorithms," Journal of Spacecraft and Rockets, vol. 36, no. 6 , pp. 859-865, 2002.

[14] G. Rauwolf and V. Coverstone-Carroll, "Near-optimal lowthrust orbit transfer generated by a genetic algorithms," Journal of Spacecraft and Rockets, vol. 33, no. 6, pp. 859-862, 1996.

[15] O. Abdelkhalik and D. Mortari, "Orbit design for ground surveillance using genetic algorithms," Journal of Guidance, Control, and Dynamics, vol. 29, pp. 1231-1235, 2006.

[16] M. Vasile and M. Locatelli, "A hybrid multiagent approach for global trajectory optimization," Journal of Global Optimization, vol. 44, no. 4, pp. 461-479, 2009.

[17] P. Gurfil and N. J. Kasdin, "Niching genetic algorithmsbased characterization of geocentric orbits in the $3 \mathrm{D}$ elliptic 
restricted three- body problem," Computer Methods in Applied Mechanics and Engineering, vol. 191, no. 49-50, pp. 5673-5696, 2002.

[18] D. A. Vallado, Fundamentals of Astrodynamics and Applications, Microcosm Press/Kluwer Academic Publishers, Dordrecht, The Netherlands, 2nd edition, 2004.

[19] P. Fortescue, J. Stark, and G. Swinerd, Spacecraft Systems Engineering, John Wiley \& Sons, New York, NY, USA, 3rd edition, 2003.

[20] D. Mortari, M. P. Wilkins, and C. Bruccoleri, "On sunsynchronous orbits and associated constellations," in Proceedings of the 6th Conference on Dynamics and Control of Systems and Structures in Space (DCSSS '04), Riomaggiore, Italy, July 2004. 

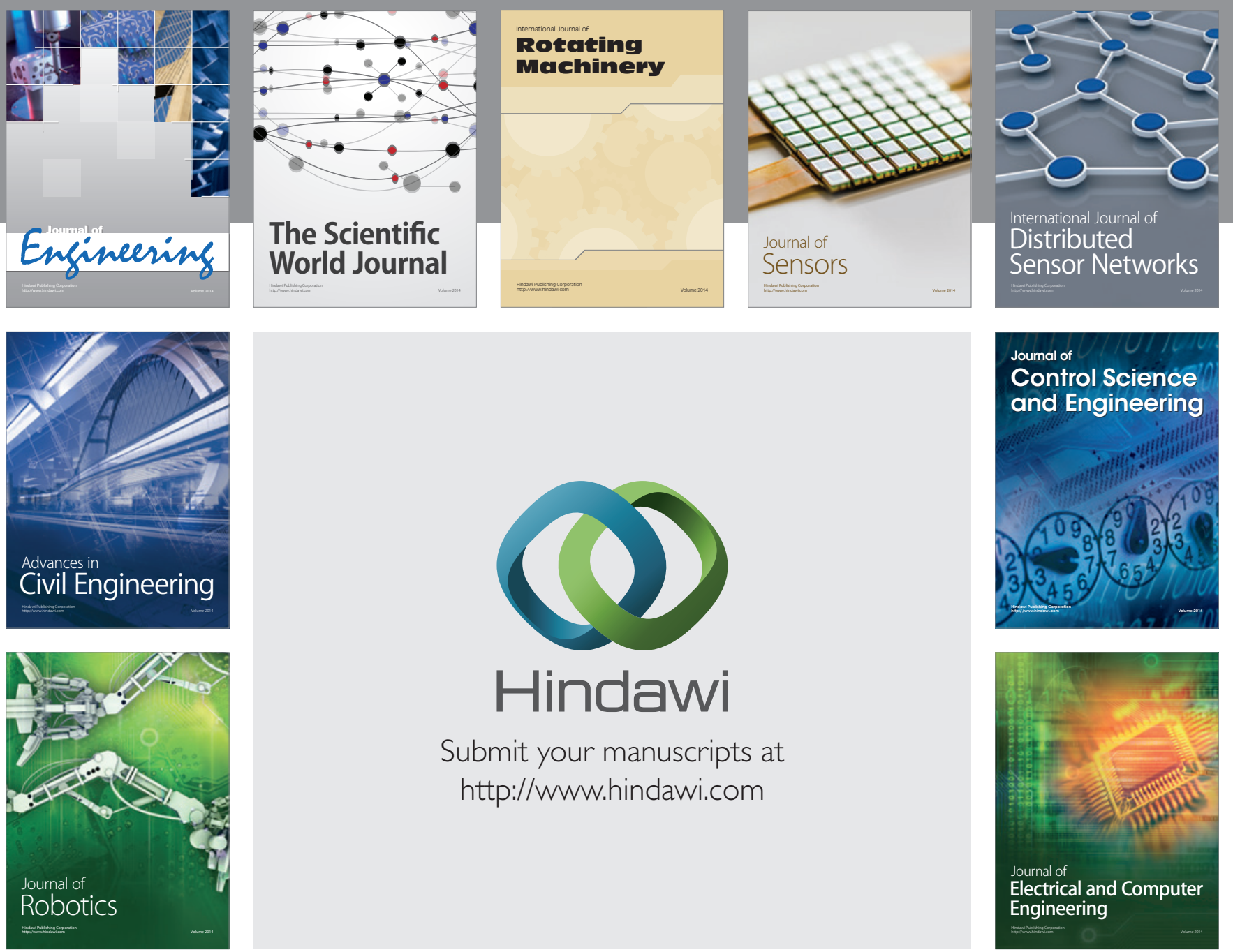

Submit your manuscripts at

http://www.hindawi.com
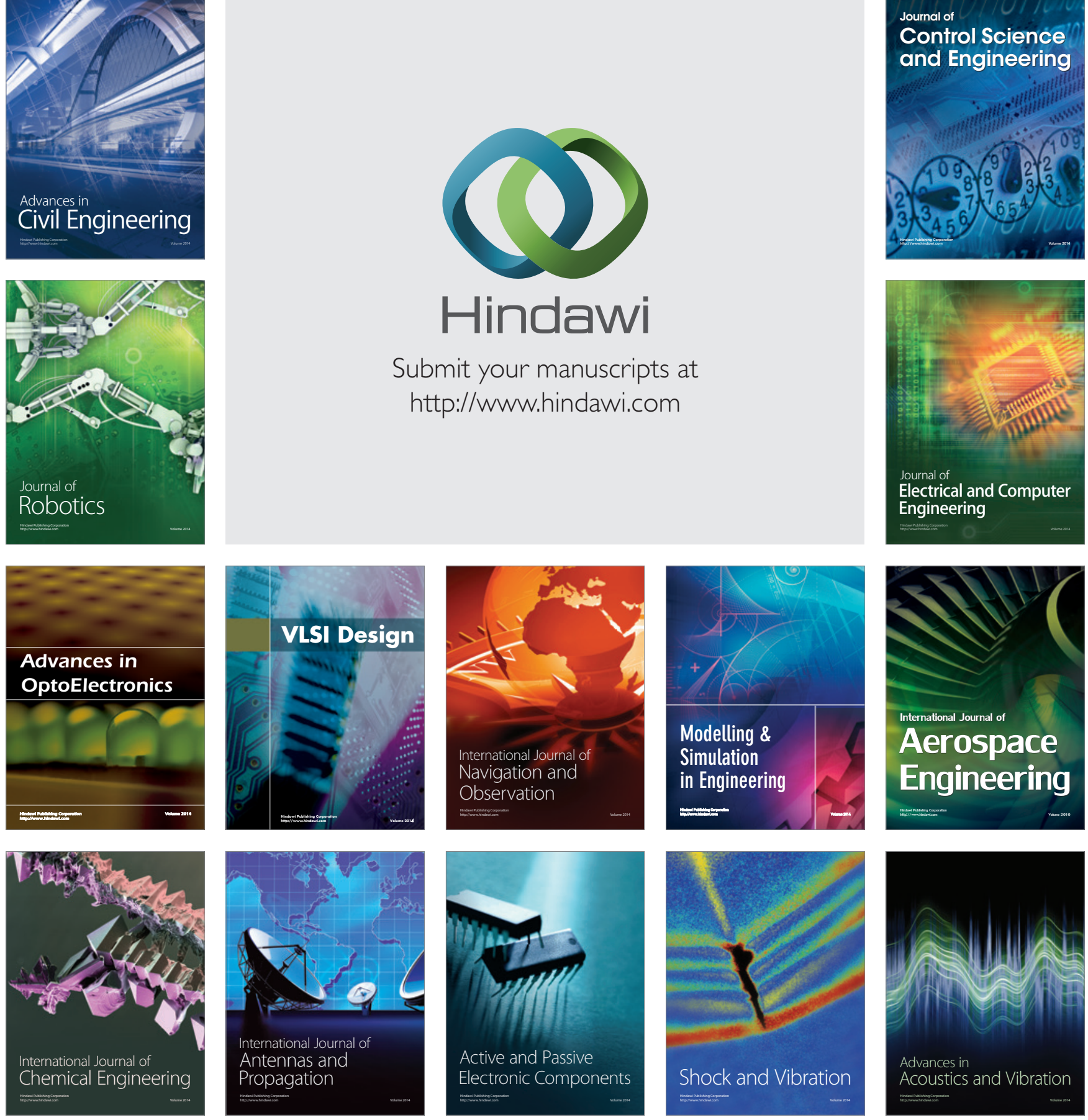